Research Article

\title{
Fuzzy Social Force Model for Pedestrian Evacuation under View-Limited Condition
}

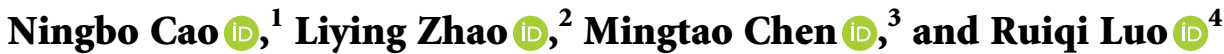 \\ ${ }^{1}$ College of Transportation Engineering, Chang'an University, Shanxi, Xi'an, China \\ ${ }^{2}$ School of Economics and Management, Xi'an University of Technology, Shanxi, Xi'an, China \\ ${ }^{3}$ Hualan Design \& Consulting Group, Guangxi, Nanning, China \\ ${ }^{4}$ North China Municipal Engineering Design \& Research Institute Co. Ltd., Tianjin, China \\ Correspondence should be addressed to Liying Zhao; zhaoliying11@126.com
}

Received 3 June 2020; Revised 14 October 2020; Accepted 27 October 2020; Published 16 November 2020

Academic Editor: Georgios I. Giannopoulos

Copyright $\odot 2020$ Ningbo Cao et al. This is an open access article distributed under the Creative Commons Attribution License, which permits unrestricted use, distribution, and reproduction in any medium, provided the original work is properly cited.

\begin{abstract}
Pedestrian evacuation dynamics in a classroom is always a complex process influenced by many fuzzy factors. It is very difficult and inappropriate to quantify the impact of these fuzzy factors by using the mathematical formula. Existing microscopic simulation models have made many efforts to use accurate mathematical method to model the fuzzy interaction behaviors between pedestrians under the view-limited condition. This study tries to fill this gap by establishing a microscopic simulation model which can represent the fuzzy behaviors of pedestrians under view-limited condition. The developed fuzzy social force model (FSFM) combines fuzzy logic into conventional social force model (SFM). Different from existing models and applications, FSFM adopts fuzzy sets and membership functions to describe the pedestrian evacuation process. Seven fuzzy sets are defined for this process, such as stop/go, moving direction, desired force, force from obstacles, force from pedestrian, force from indicators, and acceleration. Membership function of each input factor is calibrated based on the observed data. Model performance is verified by comparing speed distribution, velocity-density relationship, and results of simulation and observation evacuation time. Besides, the proposed model is applied to assess the number and space distribution of exit indicators and stickers. By comparing simulation results with existing models, the paper concludes that FSFM is able to well reproduce pedestrian movement dynamics in real world under view-limited condition.
\end{abstract}

\section{Introduction}

Pedestrian microscopic simulation model is a convenient and effective tool to evaluate the effectiveness of new facilities or designs. It can reproduce many pedestrian evacuation characteristics and help designers to determine the best solution. For example, in the field of architecture, evacuation characteristics of pedestrians can help to design a safer environment and better evacuation areas. Multiple simulations of different scenarios and facilities can help pedestrians find out how to evacuate quickly and effectively.

Generally speaking, pedestrian simulation model is the key step when analyzing the overall emergency evacuation characteristics of pedestrians. The current simulation models are usually split into two classes: macromodel and micromodel $[1,2]$. Macromodels consider pedestrians as a whole and describe them with uniform and continuous fluid or gas. The interactions between pedestrians obey the law of mass conservation. Henderson [3] used the concept of gas/ fluid dynamics in the pedestrian evacuation dynamics for the first time. Considering the shortcomings of the LWR model, Hughes [4] extended the model by developing a two-dimensional macromodel, and the improved model can be used to simulate the emergency evacuation of large-scale pedestrians. On the basis of the Hughes model, Mahato et al. [5] further improved the simulation effect by combining the optimal control method. In order to model the route choice behavior of pedestrians, Hoogendoorn et al. [6] put forward a macro continuous model. By taking the pedestrians flow as a whole, the simulation calculation required of the 
macromodel is smaller. Besides, the demand for computer hardware is also low. But the macromodel cannot describe the microscopic behaviors of pedestrians and differences between individuals. Furthermore, they cannot be used to represent complex interactions between pedestrians in detail.

Cellular automata model belongs to discrete and spatial model. Cellular automata (CA) model adopts movement rules to update pedestrian location and speed in every simulation step. In the CA model, different evacuation scenes of the pedestrian are gridded into a series of cells, and pedestrians can move to 8 cells around its current cell. Because the cellular automata model can reproduce many typical evacuation phenomena effectively, such as "lane formation", "clogging at exit doors" and "faster is slower," it obtains a more widespread application. In recent years, floor field (FF) model which involves a static and a dynamic floor field to control pedestrian movements is put forward to improve the cellular automata model [7]. Later, many scholars further extended and improved this model, for example, vector floor field (VFF) model and FF model with hexagonal cells were successfully established to simulate pedestrian movements in complex scenarios $[8,9]$. Based on the CA model, Tang et al. [10] put forward an improved CA model to simulate the movements of pedestrians in a highspeed railway station. Zhou et al. [11] analyzed how the guidance information affects the movement direction of pedestrians by adopting the CA model. Feliciani and Nishinari [12] pointed out pedestrians have different behavior characteristics when pedestrian density is high, so they developed a modified CA model to simulate the pedestrian evacuation in this condition. However, because pedestrian behaviors are random and fuzzy, CA model was combined with fuzzy logic model. In fact, the application of the fuzzy logic model in pedestrian simulation has been an ongoing concern. Pedestrians have different personality and physical state and the familiarity with the environment is different. Due to these features, Fu et al. [13] adopt a fuzzy logic model to deal with the conflicts between pedestrians. On the basis of the fuzzy logic model, Zhou et al. [14] developed a pedestrian simulation model based on fuzzy logic approach to describe crowd evacuation behaviors, and they consider the effect of assailants. Recently, the fuzzy logic model begins to be combined into CA model. Based on the idea of fuzzy logic and cellular automata, Liu et al. [15] developed a CA model to simulate the pedestrian dynamic evacuation in the room where they have several exits. In the evacuation scene, the information received by pedestrians is uncertain and random, and fuzzy logic model can deal with this problem exactly right. So, Yang et al. [16] adopt the fuzzy logic to describe unclear qualitative knowledge and experience which are hard to quantify, and combine it into CA model. To some extent, fuzzy logic model makes up for the defects of cellular automata model. But because of the inherent characteristics and settings of discrete models, simulation results are relatively rougher than continuous microscopic simulation models. Furthermore, in these discrete models, behavior characteristics, for example, fuzzy stop/go decisions of pedestrian, fuzzy judgment of indication signs in the environment, willingness of movements, as well as walking in crowd, have seldom been considered.

Compared to the above simulation models, SFM is the most reliable and fast microscopic model. The social force model not only has the advantages of the above simulation models, but also can model the behaviors of pedestrians in detail. On the basis of Newton's second law, Helbing and Moln'ar [17] quantified the interactions between pedestrians and obstacles into physical forces. They pointed out that pedestrian behaviors are determined by the combined forces of internal and external forces. The influences of external factors on pedestrian behaviors can be expressed as forces according to the form of proposed social force. In recent years, social force model has become the research focus of pedestrian simulation models. Xi et al. [18] found that the field of pedestrian vision is limited, which may lead collisions to occur. Yuen and Lee [19] found that pedestrians could decide how to go ahead according to the speed difference with other pedestrians around them. They established a modified social force model to represent the overtaking behavior. Lakoba et al. [20] added a repulsive force between pedestrians to basic social force model and solve the phenomenon of pedestrian overlap occurring in simulation. Parisi et al. [21] improved the social force model and obtained a velocity-density relation which is more accurate and realistic. Xu and Duh [22] simulated the behaviors of walking in group based on social force model and pointed out that walking in group is not conducive to pedestrian evacuation. Johansson et al. [23] aimed at modeling the waiting behavior which occurs due to the influences of external factors. They analyzed the influence of waiting behavior on pedestrian evacuation. Han and Liu [24] added an information transmission mechanism to the social force model for pedestrians who were unfamiliar with the environment. The above research studies focus on modifying the shortcomings of the initial social force model by adding other forces. However, as these models basically simulate the fuzzy cognitions, so calibration is very challenging. Another key constraint of such models is high computational complexity due to a large number of inputs and complicated utility functions in decision-making processes.

Generally speaking, under view-limited condition, pedestrians cannot accurately determine the exact locations of obstacles, indicators, and other facilities. For example, they can only tell that one obstacle is in front of them on the left, but they cannot tell the exact location. Or, pedestrians can tell that the other pedestrian speed is fast, but they cannot tell the exact value of speed. In practice, pedestrians determine their own movement behavior according to these fuzzy judgments rather than accurate information. Fuzzy logic model can solve these problems exactly right. Considering the fuzzy characteristics of pedestrian behaviors, this paper combines fuzzy logic model into SFM to represent these fuzzy and uncertainty behaviors. The developed model translates the social forces into linguistic expressions rules and fuzzy sets. And, the idea of social force model is used to calculate the acceleration of pedestrian at every simulation step. This paper establishes a model based on the fuzzy logic 
model and social force model to solve the pedestrian dynamic evacuation problem under view-limited condition. The proposed model can help designers to improve the evacuation efficiency by considering the limited visibility, indicator settings, and so on. Compared to other simulation models, it can also reproduce the same evacuation behaviors and phenomenon with an easier interpretation of pedestrian fuzzy and uncertainty behaviors. Moreover, linguistic rules contain all the forces involved in the SFM. They are more suitable to represent environment and responses which are uncertain and fuzzy. These linguistic rules are able to be adopted to model pedestrian decision-making process, such as stop/go behavior, following willing, and walking according to indicators. In this way, the pedestrian fuzzy behaviors under view-limited condition can be modeled in a very clear and straightforward way. For example, pedestrians may not sure where the exit is, so they determine their fuzzy movement behaviors. Moreover, different behaviors of pedestrian can be modeled by more than one fuzzy set, so this proposed model is easy to extend and add new rules.

\section{Initial Social Force Model}

The initial social force model (ISFM) was proposed by Helbing and Molnár [17], and the main equation of ISFM is shown in the following equation:

$$
\vec{f}_{\alpha}(t)=m \frac{\mathrm{d} \vec{v}_{\alpha}(t)}{\mathrm{d} t}=\vec{f}_{\alpha}^{0}(t)+\sum_{\beta} \vec{f}_{\alpha \beta}(t)+\sum_{o} \vec{f}_{o \alpha}(t)+\vec{\varepsilon}_{\alpha}(t),
$$

where $\vec{\varepsilon}_{\alpha}(t)$ is expressed as follows:

$$
\vec{\varepsilon}_{\alpha}(t)=\left\langle\vec{e}_{\alpha}(t), \vec{f}_{\alpha}(t)\right\rangle X \vec{e}_{\alpha}^{\text {norm }}(t)
$$

in which $\vec{f}_{\alpha}(t)$ is the resultant of forces of pedestrian $\alpha$ at moment $t ; m$ is the mass of the pedestrian, and its value is assumed to be unit mass; $\vec{f}_{\alpha}^{0}(t)$ is the driving force; $\vec{f}_{o \alpha}(t)$ is the force from obstacles; $\vec{f}_{\alpha \beta}(t)$ is the force from other pedestrians; $\vec{\varepsilon}_{\alpha}(t)$ represents the diverse random behaviors that cannot be predicted; $\vec{e}_{\alpha}(t)$ is the unit vector of $\vec{v}_{\alpha}(t)$; $\vec{e}_{\alpha}^{\text {norm }}(t)$ is the vertical unit vector of $\vec{e}_{\alpha}(t)$; and $\vec{v}_{\alpha}(t)$ is the velocity of pedestrian $\alpha$ at moment $t, X \sim N(0,1)$ :

$$
\begin{aligned}
& \vec{f}_{\alpha}^{0}(t)=m \frac{v_{\alpha}^{0}(t) \vec{e}_{\alpha}^{0}(t)-\vec{v}_{\alpha}(t)}{\tau_{\alpha}}, \\
& \vec{e}_{\alpha}^{0}(t)=\frac{\vec{p}_{\alpha}^{d}(t)-\vec{p}_{\alpha}(t)}{\left|\vec{p}_{\alpha}^{d}(t)-\vec{p}_{\alpha}(t)\right|},
\end{aligned}
$$

where $v_{\alpha}^{0}(t)$ is the desired velocity of pedestrian $\alpha$ at moment $t ; \vec{e}_{\alpha}^{0}(t)$ is the unit vector in the desired velocity direction; $\vec{p}_{\alpha}^{d}(t)$ is the coordinate of pedestrian desired destination; $\vec{p}_{\alpha}(t)$ is the coordinate of pedestrian position; $\tau_{\alpha}$ is the relaxation time of pedestrian. $\vec{f}_{\alpha \beta}(t)$ is expressed as follows:

$$
\begin{aligned}
& \vec{f}_{\alpha \beta}(t)=\vec{f}_{\alpha \beta}^{\mathrm{soc}}(t)+\vec{f}_{\alpha \beta}^{\mathrm{PH}}(t), \\
& \vec{f}_{\alpha \beta}^{\mathrm{soc}}(t)=A_{\alpha \beta} \exp \left[\frac{r_{\alpha \beta}(t)-\mathrm{d}_{\alpha \beta}(t)}{B_{\alpha \beta}}\right] \vec{n}_{\alpha \beta}(t) F_{\alpha \beta}(t), \\
& \vec{n}_{\alpha \beta}(t)=\left(n_{\alpha \beta}^{1}, n_{\alpha \beta}^{2}\right)=\frac{\vec{p}_{\alpha}(t)-\vec{p}_{\beta}(t)}{\mathrm{d}_{\alpha \beta}(t)}, \\
& \vec{f}_{\alpha \beta}^{\mathrm{PH}}(t)=K g\left\{r_{\alpha \beta}(t)-\mathrm{d}_{\alpha \beta}(t)\right\} \vec{n}_{\alpha \beta}(t)+k g\left\{r_{\alpha \beta}(t)-\mathrm{d}_{\alpha \beta}(t)\right\} \Delta v_{\alpha \beta}^{t}(t) \vec{t}_{\alpha \beta}(t) \text {, } \\
& \vec{t}_{\alpha \beta}(t)=\left(-n_{\alpha \beta}^{2}(t), n_{\alpha \beta}^{1}(t)\right) \text {, } \\
& \Delta v_{\alpha \beta}^{t}(t)=\left\{\vec{v}_{\alpha}(t)-\vec{v}_{\beta}(t)\right\} * \vec{t}_{\alpha \beta}(t), \\
& g(x)= \begin{cases}x, & x>0, \\
0, & \text { otherwise }\end{cases}
\end{aligned}
$$

where $\vec{f}_{\alpha \beta}^{\text {soc }}(t)$ and $\vec{f}_{\alpha \beta}^{\mathrm{PH}}(t)$ are the sociopsychological and physical forces at moment $t$, respectively; $A_{\alpha \beta}$ is the strength coefficient of $\vec{f}_{\alpha \beta}(t) ; B_{\alpha \beta}$ is the reaction distance coefficient of $\vec{f}_{\alpha \beta}(t) ; r_{\alpha \beta}(t)$ is the sum of the radiuses of pedestrian $\alpha$ and $\beta$ at moment $t ; d_{\alpha \beta}(t)$ is the distance between the centers of pedestrians $\alpha$ and $\beta$ at moment $t ; \vec{n}_{\alpha \beta}(t)$ is the vector pointing from pedestrian $\alpha$ to $\beta ; F_{\alpha \beta}(t)$ is the anisotropic factor; $\Delta v_{\alpha \beta}^{t}(t)$ is the velocity difference between pedestrians 
$\alpha$ to $\beta$ in the tangent direction; $\vec{t}_{\alpha \beta}(t)$ is the vertical vector of $\vec{n}_{\alpha \beta}(t) ; K$ and $k$ are very large constants.

Different values of $F_{\alpha \beta}(t)$ represent that pedestrians in front/behind of him have different influences on the subject pedestrian. Pedestrians in the vision of subject pedestrian have the biggest influences, and pedestrians in the behind of subject pedestrian have minimal influences. It can be calculated by the following formula:

$$
\begin{aligned}
F_{\alpha \beta}(t) & =\lambda_{\alpha}+\left(1-\lambda_{\alpha}\right) \frac{1+\cos \left(\varphi_{\alpha \beta}(t)\right)}{2}, \\
\cos \left(\varphi_{\alpha \beta}(t)\right) & =-\vec{n}_{\alpha \beta}(t) \vec{e}_{\alpha}(t) .
\end{aligned}
$$

When $\lambda_{\alpha}=1, F_{\alpha \beta}(t)=1$, which means that pedestrians around the subject pedestrian have the same influence on him. When $0 \leq \lambda_{\alpha}<1$, which means that pedestrians in front of subject pedestrian have bigger influence, and $\varphi_{\alpha \beta}(t)$ is the angle between $\vec{v}_{\alpha}(t)$ and $\vec{n}_{\alpha \beta}(t)$. $\vec{f}_{o \alpha}(t)$ is expressed as follows:

$$
\begin{aligned}
\vec{f}_{o \alpha}(t)= & \vec{f}_{o \alpha}^{\mathrm{soc}}(t)+\vec{f}_{o \alpha}^{\mathrm{PH}}(t), \\
\vec{f}_{o \alpha}^{\mathrm{soc}}(t)= & A_{o \alpha} \exp \left[\frac{\left\{r_{o \alpha}(t)-d_{o \alpha}(t)\right\}}{B_{o \alpha}}\right] \vec{n}_{o \alpha}(t), \\
\vec{f}_{o \alpha}^{\mathrm{PH}}(t)= & K g\left\{r_{o \alpha}(t)-d_{o \alpha}(t)\right\} \vec{n}_{o \alpha}(t) \\
& -k g\left\{r_{o \alpha}(t)-d_{o \alpha}(t)\right\} \Delta v_{o \alpha}^{t}(t) \vec{t}_{o \alpha}(t), \\
\Delta \vec{v}_{o \alpha}^{t}(t)= & \vec{v}_{\alpha}(t) * \vec{t}_{o \alpha}(t),
\end{aligned}
$$

where $\vec{f}_{o \alpha}^{\mathrm{soc}}(t)$ and $\vec{f}_{o \alpha}^{\mathrm{PH}}(t)$ are the sociopsychological and physical forces between pedestrian $\alpha$ and obstacle $o$, respectively; $\vec{n}_{\rho \alpha}(t)$ is the vector pointing from pedestrian $\alpha$ to obstacle $o ; \vec{t}_{o \alpha}(t)$ is vertical vector of $\vec{n}_{o \alpha}(t)$.

\section{Fuzzy Social Force Model Development}

3.1. Fuzzy Logic. Zadeh [25] put forward the fuzzy logic model which uses fuzzy sets to study fuzzy thinking, language forms, and their laws. In this progress, degrees of truth are used to explain the vagueness in propositions. Generally speaking, fuzzy logic is expressed in the linguistic rules which are used as "IF variable input IS fuzzy set THEN variable output IS fuzzy set”. It is processed as follows by fuzzy inference systems:

(i) Fuzzification: this step converts crisp data (real numbers) to fuzzy data, the degree of membership of the crisp input to predefined fuzzy sets

(ii) Inference: you can combine the inputs by using logical, fuzzy rules to determine the degree to which each rule is true

(iii) Defuzzification: defuzzification is required when you want a crisp number as output from a fuzzy system
In order to describe the degrees of truth, fuzzy variable should contain several fuzzy sets. One set has one membership function. The arguments of the function are crisp values, and the calculated value according to the function is between 0 and 1, which represents the degree of truth. Fuzzy rules make these incomprehensible factors easier to be understood, parametrized, and extended. The fuzzy inference system uses fuzzy theory as the main calculation tool to implement complex nonlinear mapping. Its input and output are crisp values, so it has wide application prospects. Dubois and Prade [26] indicated that fuzzy is very common. So, we can use similarity, preference, and uncertainty to explain the membership function. Similarity is used in fuzzy cluster set analysis and related systems. Preference is mostly used in choice decision. Uncertainty is the degree of ambiguity and possibility and has been adopted by expert systems and AI. Similarity, preference and uncertainty are not mutually exclusive, and they are combined into a fuzzy inference system to indicate the objective. Fuzzy logic can descript the preference and uncertainty behaviors of pedestrians. Nasir et al. [27] combined fuzzy rules into agentbased method to simulate pedestrian movements. They indicated that the parameters of developed fuzzy rules are calibrated by using real data, and the model has good performance. Then, they combined fuzzy rules into social force model and used SFM output as input to the fuzzy system, and it contains 216 rules. This modified model is used to derive linguistic fuzzy rules, which can explain the preference and uncertainty behaviors of pedestrians and need less works to fine tune. Besides, Nasir et al. [28] indicated that it works better than the traditional social force model when trained with real data using machine learning techniques.

3.2. Cognition System of Individual Pedestrian. The proposed fuzzy social force model adopts fuzzy logic to control pedestrian evacuation. Fuzzy sets and rules are defined for pedestrian behaviors influenced by different environment and individual attributes. The factors from environment and individual attributes are analyzed to determine the key factors influencing the decision-making process of pedestrians, as shown in Figure 1. This decision-making process may occur at several levels. For instance, decisions are made according to the comprehensive consideration of environment situations, psychology reasoning, and physiology movement. Factors of each process are shown in Figure 1.

As shown in Figure 2, this paper uses the fuzzy logic to model the process of cognition. The influencing factors of pedestrian behaviors are modeled as fuzzy inputs, and the moving decisions are modeled as fuzzy outputs. For example, pedestrian current velocity, position, and whether he/ she follows group determine his status in the next time step. Perception contains exit location, whether safety exit indicator/exit stickers can be seen, neighboring pedestrians, and obstacle (wall, tables, and chairs). Intention contains desired velocity and position. Attitude contains whether he/she is 


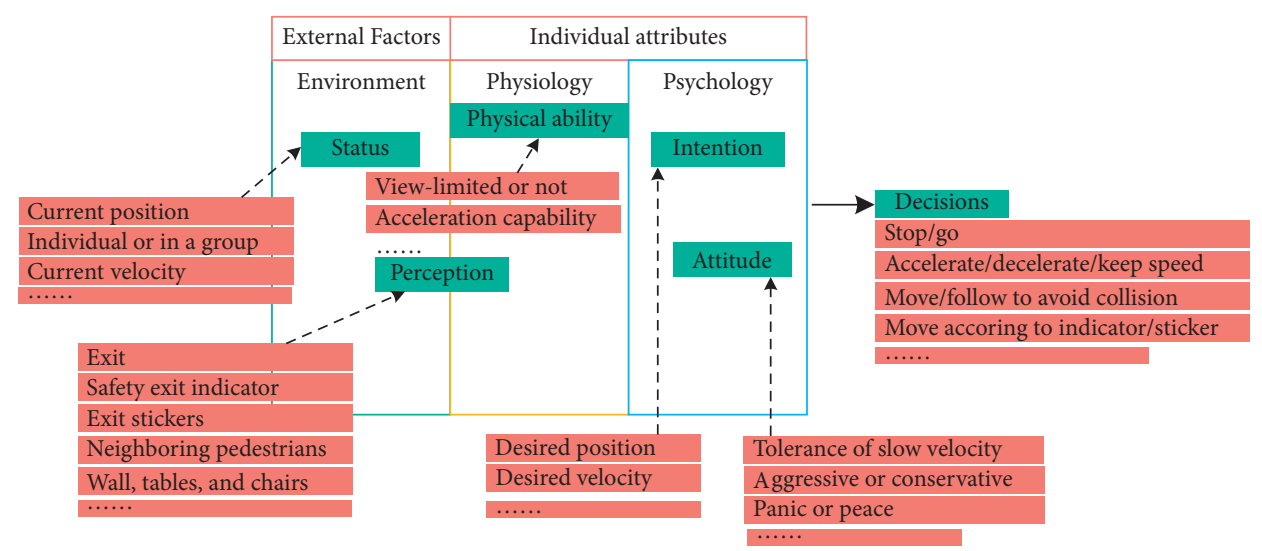

FIGURE 1: Decision-making process of pedestrian.

aggressive or conservative and whether he/she feels panic or peace. Aggressive and conservative vary among individuals, and they are the inherent property. Combining the above information, pedestrian makes decisions, for example, whether he/she should go or stop, which position he/she should move toward, and whether he/she should move according to safety exit indicator/exit stickers.

3.3. Fuzzy Sets and Rules. As shown in Table 1, seven sets of fuzzy rules named as $F_{1}$ to $F_{7}$ are introduced to describe pedestrian behaviors. These seven sets are used to simulate pedestrian stop/go decisions, moving direction, and force acted on pedestrians, respectively. Each fuzzy set includes one or more subsets that represent different influencing factors. For instance, moving direction of each pedestrian is modeled by three subsets, such as exit, indicator, and random behavior. All these input and output factors adopt linguistic terms to explain, because this paper focuses on developing a FSFM method innovatively, so a relatively simple simulation scene (classroom) is assumed to reduce the difficulty of model building.

Fuzzy rules are defined based on the psychological reaction about the external environment of pedestrian. For example, fuzzy set $F_{3-1}$ rule: if Relaxing capability is Fast and Velocity difference with desired velocity is Very Slow, then Fuzzy desired force $\vec{f}_{\alpha}(t)$ is Strong positive. Fuzzy set $F_{4-1}$ rule: if Distance to neighboring desk is Far, Distance to neighboring chair is Far, and Distance to neighboring wall is Far, then Fuzzy force from obstacles $\vec{f}_{o \alpha}(t)$ is Zero.

Fuzzy sets $F_{5}$ and $F_{6}$ model pedestrian behaviors affected by other pedestrians and indicators around them. In fuzzy set $F_{2}$, pedestrian movement direction is divided into 8 possible areas, as shown in Figure 3. The desired direction is defined as front direction, and it has the most priority. The second priority is right front considering the habit that pedestrians prefer to walk on the right followed by right, left front; left; right back; left back; and back. If space ahead and lateral space along prior moving directions are occupied by other pedestrians, the directions with the next level of priority will be searched.

\subsection{Combining Fuzzy Sets in SFM}

3.4.1. Fuzzy Desired Force. Pedestrian wants to move at the desired speed in the desired direction because they prefer to keep themselves comfortable. In the classroom environment, the desired direction points to the exit door or indicators (exit indicator and sticker), and the desired speed is the average speed of pedestrians. However, the direction and speed may be compromised by other pedestrians and obstacles. For example, they have to change the desired direction when the desired direction is occupied, and they have to reduce their speed when they are blocked by obstacles or pedestrians.

In the ISFM, the desired force is determined by relaxing time and velocity difference with desired velocity, so we separate each of these factors into two subsets of rules, one to treat the relaxing capability and the other to treat the velocity difference with desired velocity. Fuzzy rules are created based on ISFM.

According to formula (3), for example, if relaxing time is small, the desired force will be large. If the velocity difference with desired velocity is small, the desired force will be small. Moreover, if relaxing time is small and velocity difference with desired velocity is large, the desired force will be very large; if relaxing time is large and velocity difference with desired velocity is large, the desired force will be medium; if relaxing time is large and velocity difference with desired velocity is small, the desired force will be very small, so we set a fuzzy set $F_{3}$ to model this process. $F_{3}$ has one subset $F_{3-1}$. Its inputs contain Relaxing capacity (classified according to relaxing time) and Velocity difference with desired velocity.

According to different combinations of relaxing time and velocity difference, we can define several fuzzy set $F_{3-1}$ rules. The fuzzy logic uses the linguistic rules to model, and its form is "IF variable input IS fuzzy set THEN variable output IS fuzzy set".

For example, fuzzy set $F_{3-1}$ rule no. 1: if Relaxing capability is Fast and Velocity difference with desired velocity is Very Slow, then Fuzzy desired force is Strong positive; fuzzy set $F_{3-1}$ rule no. 2: if Relaxing capability is Fast and Velocity difference with desired velocity is Very Fast, then Fuzzy desired force is Strong negative. 


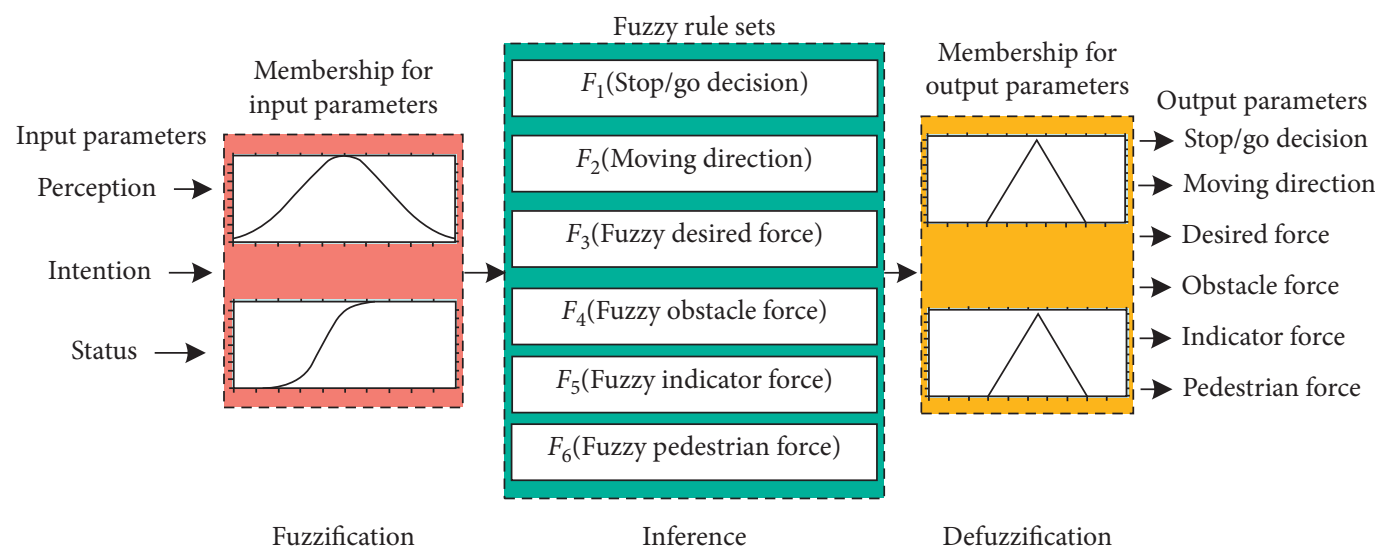

FIgURE 2: Operation diagram of FSFM.

TABLE 1: Fuzzy sets and terms of the developed FSFM.

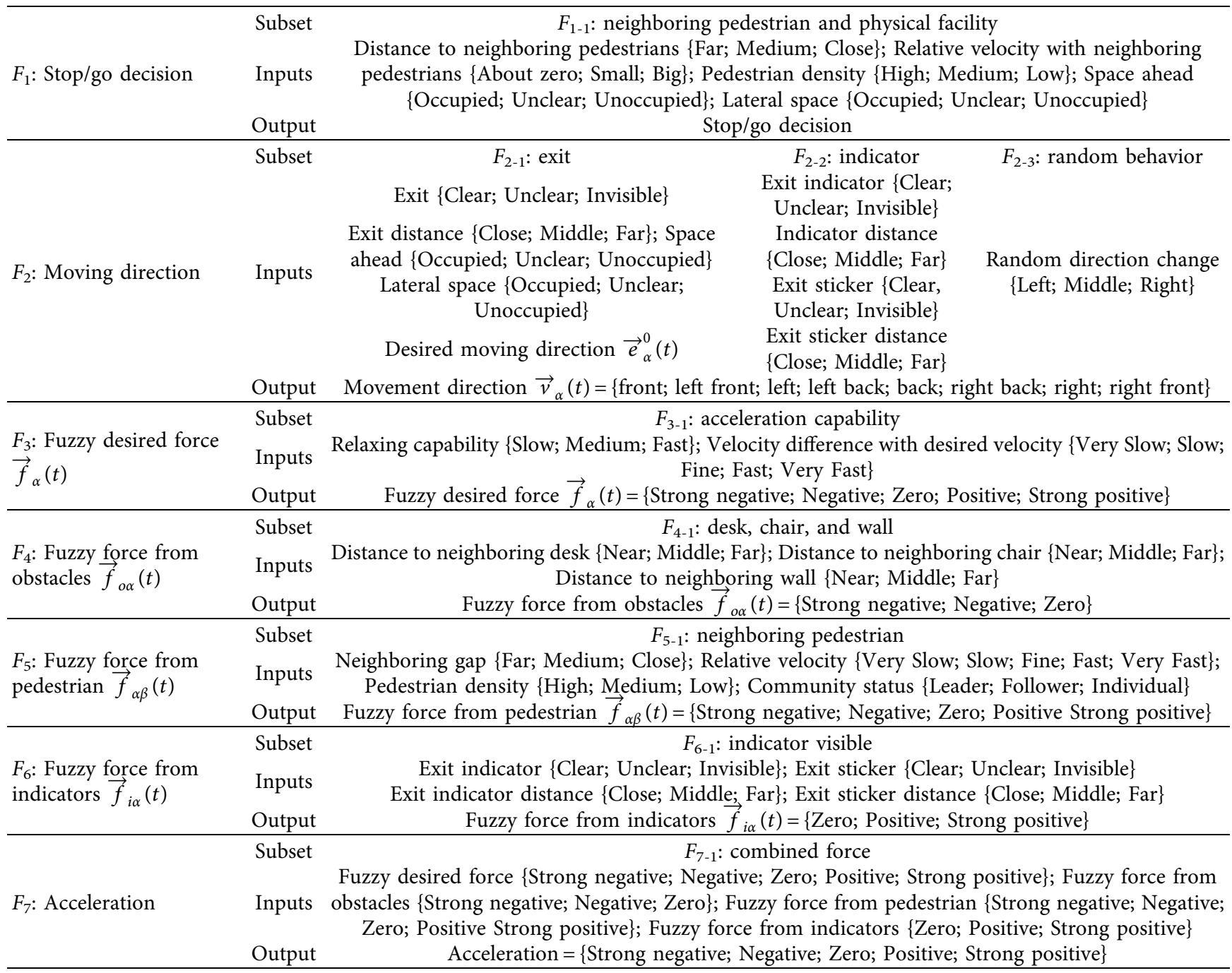

Because Relaxing time and Velocity difference can be combined randomly, there are many kinds of combinations. That is, there are many rules for one fuzzy set, so we only take several as examples to explain. In the simulation, all the rules will exist.
According to the distribution law of relaxing time/velocity difference with desired velocity, the paper selects and calibrates the membership function of each fuzzy set. The detailed methods on the selection of parameters and the determination of membership functions will be introduced 


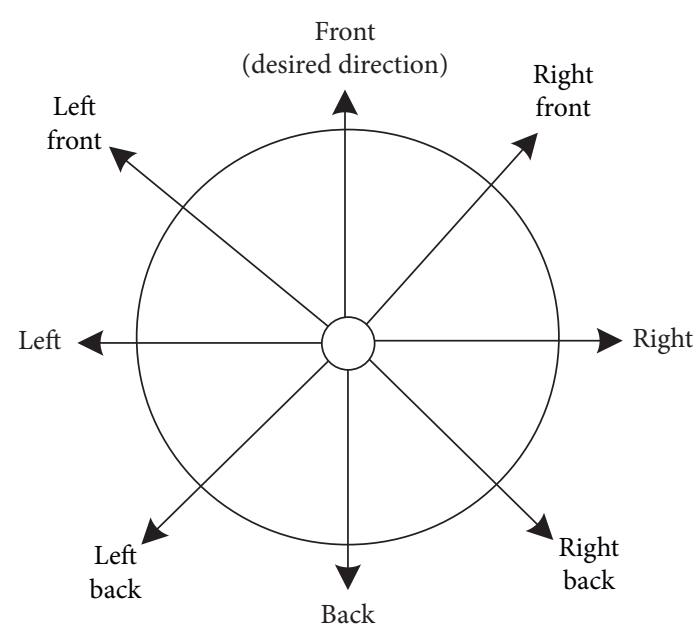

Figure 3: Possible directions of movement.

in Section 4. And this section only explains the meaning of each fuzzy set in detail. According to the method introduced in Section 4, the input variable Relaxing capability is divided into three Gaussian sets. According to formula (27), the sets configuration is as follows: Slow set is $s=0.1$ and $c=1$; Medium set is $s=0.1$ and $c=0.7$; and Fast set is $s=0.1$ and $c=0.4$. And they can be expressed as follows:

$$
\begin{aligned}
& \operatorname{gaussmf}(x)=e^{-\left((x-1)^{2} / 2 * 0.1^{2}\right)} \\
& \operatorname{gaussmf}(x)=e^{-\left((x-0.7)^{2} / 2 * 0.1^{2}\right)} \\
& \operatorname{gaussmf}(x)=e^{-\left((x-0.4)^{2} / 2 * 0.1^{2}\right)}
\end{aligned}
$$

The input variable Velocity difference with desired velocity is divided into five Gaussian sets. These parameters are put into formula (27) in the same way. The sets configuration is as follows: Very Slow set is $s=0.3$ and $c=-2$; Slow set is $s=0.3$ and $c=-1$; Fine set is $s=0.3$ and $c=0$; Fast set is $s=0.3$ and $c=1$; and Very Fast set is $s=0.3$ and $c=2$. The output variable Fuzzy desired force $\vec{f}_{\alpha}(t)$ is divided into five triangular sets to represent the influence degree. The sets configuration is as follows: Strong negative set is $a=-3, b=-2$, and $c=-1$, and they can be expressed as formula (21); Negative set is $a=-2$, $b=-1$, and $c=0$; Zero set is $a=-1, b=0$, and $c=1$; Positive set is $a=0, b=1$, and $c=2$; Strong positive set is $a=1, b=2$, and $c=3$. These parameters are all put into formula (25) in the same way. All the processes are the same, so we will not describe it in detail in the following sections:

$$
\text { triangmf }(x)= \begin{cases}0, & \text { if }(x<-3) \|(x>-1), \\ 1, & \text { if }(x=-2), \\ (x+3) /(-2+3), & \text { if }(x<-2), \\ (-1-x) /(-1+2), & \text { otherwise. }\end{cases}
$$

3.4.2. Fuzzy Force from Obstacles. Pedestrians try to avoid obstacles to keep themselves safe. In the classroom environment, obstacles (desk, chair, and wall) prevent pedestrians from moving. So the force from obstacles is negative. In the ISFM, the force from obstacles is determined by the distance to obstacles, so we separate each of these factors into three subsets of rules, one to treat the distance to neighboring desk, other to treat the distance to neighboring chair, and the other to treat the distance to neighboring wall. Fuzzy rules are created based on ISFM.

According to formula (15), for example, if distance to obstacle is small, the force from obstacles will be large. And if the distance to obstacle is large, the desired force will be small. However, because there are many kinds of obstacles in the classroom, such as tables, chairs, and walls, pedestrians may face several obstacles at one moment, so we set a fuzzy set $F_{4}$ to model this process. $F_{4}$ has one subset $F_{4-1}$. Its inputs contain three factors: Distance to neighboring desk, Distance to neighboring chair, and Distance to neighboring wall. According to different combinations of these three factors, we can define several fuzzy set $F_{4-1}$ rules in the form of linguistic rules "IF variable input IS fuzzy set THEN variable output IS fuzzy set".

For example, fuzzy set $F_{4-1}$ rule no. 1: if Distance to neighboring desk is Near, Distance to neighboring chair is Near, and Distance to neighboring wall is Near, then Fuzzy force from obstacles is Strong negative; fuzzy set $F_{4-1}$ rule no. 2: if Distance to neighboring desk is Far, Distance to neighboring chair is Far, and Distance to neighboring wall is Far, then Fuzzy force from obstacles is Zero.

The same method on the selection of parameters and the determination of membership functions will be used. We also only take several as examples to explain. In the simulation, all the rules will exist. The input variable Distance to neighboring desk is divided into three sigmoidal sets. These parameters are all put into formula (26) in the same way. The sets configuration is as follows: Near set is slope $=-10$ and inflection $=0.5$; Middle set is slope $=-10$ and inflection $=1$; and Far set is slope $=-10$ and inflection $=1.5$. And they can be expressed as follows:

$$
\begin{aligned}
& \operatorname{sigmf}(x)=\frac{1.0}{\left(1.0+e^{-10 *(x-0.5)}\right)} \\
& \operatorname{sigmf}(x)=\frac{1.0}{\left(1.0+e^{-10 *(x-1)}\right)} \\
& \operatorname{sigmf}(x)=\frac{1.0}{\left(1.0+e^{-10 *(x-1.5)}\right)}
\end{aligned}
$$

The input variable Distance to neighboring chair is also divided into three sigmoidal sets. The sets configuration is as follows: Near set is slope $=-10$ and inflection $=0.5$; Middle set is slope $=-10$ and inflection $=1$; and Far set is slope $=-10$ and inflection $=1.5$. The input variable Distance to neighboring wall is also divided into three sigmoidal sets. The sets configuration is as follows: Near set is slope $=-10$ and inflection $=2$; Middle set is slope $=-10$ and inflection $=3$; and Far set is slope $=-10$ and inflection $=4$. These parameters are all put into formula (25) in the same way. The output variable Fuzzy force from obstacles $\vec{f}_{o \alpha}(t)$ is divided 
into three triangular sets to represent the influence degree. The sets configuration is as follows: Strong negative set is $a=-3, b=-2$, and $c=-1$; Negative set is $a=-2, b=-1$, and $c=0$; and Zero set is $a=-1, b=0$, and $c=1$.

3.4.3. Fuzzy Force from Pedestrian. Pedestrians try to follow pedestrians to keep themselves safe and comfortable. In the classroom, pedestrians prefer to move in group.

For example, according to formula (6), if distance to other pedestrians is small, the force from other pedestrians will be large. Under this condition, subject pedestrian has little space to walk, and they have to slow down. If the distance to other pedestrians (pedestrian gap) is large, the force from pedestrians will be small. Under this condition, subject pedestrian has enough space to walk, and they prefer to move at the desired speed. Besides, if subject pedestrian locates in the front of the crowd, and he/she is defined as a leader. Otherwise, he/she is defined as a follower. A leader receives less influences (small force) from other pedestrians. A follower receives more influences (large force) from other pedestrians. When pedestrians are in crowd, they will prefer to move at a speed which is close with other pedestrians in the crowd. So, the relative velocity with other pedestrians in the crowd is taken as an input factor. Pedestrians may determine their behaviors according to the above factors at one moment, so we set a fuzzy set $F_{5}$ to model this process. $F_{5}$ has one subset $F_{5-1} \cdot F_{5-1}$ inputs contain four factors: Neighboring gap, Relative velocity, Pedestrian density, and Community status. According to different combinations of these four factors, we can also define several fuzzy set $F_{5-1}$ rules in the form of linguistic rules "IF variable input IS fuzzy set THEN variable output IS fuzzy set".

For example, fuzzy set $F_{5-1}$ rule no.1: if Neighboring gap is Close, Relative velocity is Fine, Pedestrian density is High, and Community status is Follower, then Fuzzy force from pedestrian is Zero; fuzzy set $F_{5-1}$ rule no. 2: if Neighboring gap is Far, Relative velocity is Very Slow, Pedestrian density is Low, and Community status is Follower, then Fuzzy force from pedestrian is Strong negative.

The same method on the selection of parameters and the determination of membership functions will be used. We also only take several as examples to explain. In the simulation, all the rules will exist. These parameters are all put into the corresponding formula from formula (25) to (27) in the same way. All the processes are the same, so we will not describe it in detail. The input variable Neighboring gap is divided into three triangular sets. The sets configuration is as follows: Far set is $a=1.5, b=2.5$, and $c=3.5$; Medium set is $a=1, b=2$, and $c=3$; and Close set is $a=0.5, b=1.5$, and $c=2.5$. The input variable Relative velocity is divided into five triangular sets. The sets configuration is as follows: Very slow set is $a=-2, b=-1$, and $c=0$; Slow set is $a=-1.5$, $b=-0.5$, and $c=0.5$; Fine set is $a=-1, b=0$, and $c=1$; Fast set is $a=-0.5, b=0.5$, and $c=1.5$; and Very Fast set is $a=0$, $b=1$, and $c=2$. The input variable Pedestrian density is divided into three triangular sets. The sets configuration is as follows: Low set is $a=0, b=1$, and $c=2$; Medium set is $a=1$, $b=2$, and $c=3$; and High set is $a=2, b=3$, and $c=4$. The input variable Community status is divided into three triangular sets. The sets configuration is as follows: Leader set is $a=1, b=1.5$, and $c=2$; Follower set is $a=0.5, b=1$, and $c=1.5$; and Individual set is $a=0, b=0.5$, and $c=1$. The output variable Fuzzy force from pedestrian $\vec{f}_{\alpha \beta}(t)$ is divided into five triangular sets to represent the influence degree. The sets configuration is as follows: Strong negative set is $a=-3, b=-2$, and $c=-1$; Negative set is $a=-2, b=-1$, and $c=0$; Zero set is $a=-1, b=0$, and $c=1$; Positive set is $a=0, b=1$, and $c=2$; and Strong positive set is $a=1, b=2$, and $c=3$.

3.4.4. Fuzzy Force from Indicators. Indicators include exit indicators and stickers. When the environment is dark and vision is limited, indicators help pedestrians move towards the exit. Generally speaking, safety exit indicator is hung on the wall. A few classrooms have exit sticker posted on the ground. So, students find it difficult to move toward the exit when an emergency occurs, especially, when they have limited view in the dark environment. Reasonable indicators setting will help students evacuate quickly. So, we introduce the influences of indicators to FSMF. For example, according to the basic form of social force, indicators may have a gravitational effect on pedestrians when they can be seen. However, if they are unable to be seen, they have little influences on pedestrians. Besides, if the distance between indicator and pedestrian is large, even if the pedestrian can see the indicator, its guiding effect on pedestrian is relatively weak. It means that force from indicators is small. Moreover, if indicators can be seen clearly and the distance between indicator and pedestrian is small, the force from indicators will be very large; so, we set a fuzzy set $F_{6}$ to model this process. $F_{6}$ has one subset $F_{6-1}$. Its inputs contain Exit indicator, Exit sticker, Exit indicator distance, and Exit sticker distance. According to different combinations of these four factors, we can define several fuzzy set $F_{6-1}$ rules. The fuzzy logic uses the linguistic rules to model, and its form is "IF variable input IS fuzzy set THEN variable output IS fuzzy set”.

For example, fuzzy set $F_{6-1}$ rule no. 1: if Exit indicator is Clear, Exit sticker is Clear, Exit indicator distance is Close, and Exit sticker distance is Close, then Fuzzy force from indicators is Strong positive; fuzzy set $F_{6-1}$ rule no.2: if Exit indicator is Invisible, Exit sticker is Invisible, Exit indicator distance is Far, and Exit sticker distance is Far, then Fuzzy force from indicators is Zero.

The same method on the selection of parameters and the determination of membership functions will be used. We also only take several as examples to explain. In the simulation, all the rules will exist. These parameters are all put into the corresponding formula from formula (25) to (27) in the same way. All the processes are the same, so we will not describe it in detail. The input variable Exit indicator is divided into three triangular sets. The sets configuration is as follows: Clear set is $a=5, b=6$, and $c=7$; Unclear set is $a=6$, $b=7$, and $c=8$; and Invisible set is $a=7, b=8$, and $c=9$. The input variable Exit sticker is divided into three triangular sets. The sets configuration is as follows: Clear set is $a=2$, 
$b=3$, and $c=4$; Unclear set is $a=3, b=4$, and $c=5$; and Invisible set is $a=4, b=5$, and $c=6$. The input variable Exit indicator distance is divided into three triangular sets. The sets configuration is as follows: Close set is $a=0, b=3$, and $c=6$; Middle set is $a=3, b=6$, and $c=9$; and Far set is $a=6$, $b=9$, and $c=12$. The input variable Exit sticker distance is divided into three triangular sets. The sets configuration is as follows: Close set is $a=0, b=1.5$, and $c=3$; Middle set is $a=1.5, b=3$, and $c=4.5$; and Far set is $a=3, b=4.5$, and $c=6$. The output variable Fuzzy force from indicators $\vec{f}_{i \alpha}(t)$ is divided into triangular sets to represent the influence degree. The sets configuration is as follows: Zero set is $a=-1$, $b=0$, and $c=1$; Positive set is $a=0, b=1$, and $c=2$; and Strong positive set is $a=1, b=2$, and $c=3$.

3.4.5. Acceleration. Fuzzy desired force, fuzzy force from obstacles, fuzzy force from pedestrian, and fuzzy force from indicators drive the formation of a joint force of the subject pedestrian. These four fuzzy forces are taken as the input factors of Acceleration. For example, according to formula (1), if the resultant force is positive and large, pedestrian will accelerate at a greater acceleration; if the resultant force is negative and large, pedestrian will slow down at a greater deceleration; if the resultant force is zero, pedestrian will stop or move at a constant speed. So, we set a fuzzy set $F_{7}$ to calculate acceleration. In this way, these fuzzy forces are combined in ISFM, and fuzzy logic is combined into social force. $F_{7}$ has one subset $F_{7-1}$. Its inputs contain Fuzzy desired force, Fuzzy force from obstacles, Fuzzy force from pedestrian, and Fuzzy force from indicators. According to different combinations of these four forces, we can define several fuzzy set $F_{7-1}$ rules. The fuzzy logic uses the linguistic rules to model, and its form is "IF variable input IS fuzzy set THEN variable output IS fuzzy set”.

For example, fuzzy set $F_{7-1}$ no. 1: if Fuzzy desired force is Strong negative, Fuzzy force from obstacles is Strong negative, Fuzzy force from pedestrian is Strong negative, and Fuzzy force from indicators is Zero, then Acceleration is Strong positive (deceleration); fuzzy set $F_{7-1}$ no. 2: if Fuzzy desired force is Strong positive, Fuzzy force from obstacles is Zero, Fuzzy force from pedestrian is Strong positive, and Fuzzy force from indicators is Strong positive, then Acceleration is Strong positive (acceleration).

The same method on the selection of parameters and the determination of membership functions will be used. We also only take several as examples to explain. In the simulation, all the rules will exist. These parameters are all put into the corresponding formula from formula (25) to (27) in the same way. All the processes are the same, so we will not describe it in detail. The output variable Acceleration is divided into five triangular sets to represent the influence degree. The sets configuration is as follows: Strong negative set is $a=-1.5, b=-1$, and $c=-0.5$; Negative set is $a=-1$, $b=-0.5$, and $c=0$; Zero set is $a=-0.5, b=0$, and $c=0.5$; Positive set is $a=0, b=0.5$, and $c=1$; and Strong positive set is $a=1.5, b=1$, and $c=0.5$.

The Fuzzy Logic Toolbox of MATLAB is adopted in this paper. The results of each pedestrian at each time step are calculated according to pedestrians around, indicators, and environment. The output results will help each pedestrian update his/her velocity and position at the next time step. The simulation flowchart is as shown in Figure 4. Firstly, fuzzy set $F_{1}$ will be used to calculate his/her stop/go decision; secondly, fuzzy set $F_{2}$ helps to determine the moving direction; thirdly, the output results of $F_{2}-F_{6}$ are used as the inputs of $F_{7}$ to calculate his/her acceleration according to SFM; finally, he/she will update position and velocity based on moving direction, acceleration, and current velocity.

\section{Model Calibration, Performance, and Application}

4.1. Calibration of Membership Functions. Generally speaking, the selection and calibration of the membership function are based on the experience of researchers, but in order to obtain more accurate simulation results, the paper selects and calibrates the membership function of each fuzzy set according to the observed data, such as Relaxing capability set in fuzzy set $F_{3-1}$. They should be extracted from actual observed data of pedestrian movements. We use the calibration method of the membership function proposed by Chai et al. [29]. We will introduce the method briefly in this section. We collected the movement data of 2484 pedestrians in many classrooms. All these classrooms are $11 \mathrm{~m} * 9 \mathrm{~m}$ and have two exits and exit indicators. This paper adopts three types of membership functions, triangular, sigmoid, and Gaussian, and their functions are as follows:

$$
\operatorname{triangmf}(x)= \begin{cases}0, & \text { if }(x<a) \|(x>c), \\ 1, & \text { if }(x==b), \\ (x-a) /(b-a), & \text { if }(x<b), \\ (c-x) /(c-b), & \text { otherwise, }\end{cases}
$$

$$
\begin{aligned}
\operatorname{sigmf}(x) & =\frac{1.0}{\left(1.0+e^{\text {slope } *(x-\text { inflection })}\right)}, \\
\operatorname{gaussmf}(x) & =e^{-\left((x-c)^{2} / 2 s^{2}\right),}
\end{aligned}
$$

where $a, b, c$, slope, inflection, and $s$ are the parameters of functions.

Because these three kinds of membership functions are widely used, besides, these three models are most close to the distribution of actual observed data. The preselection process of membership function can improve the calculation efficiency. Three steps are needed to calibrate the membership function of each fuzzy factor, and this section takes the fuzzy factors (Relaxing capacity and Velocity difference with desired velocity) in one subset $F_{3-1}$ as example, as shown in Figure 5. Because there are many fuzzy factors in seven subsets, we cannot introduce all the calibration processes of all fuzzy factors in detail. So, we take relaxing capacity as an example, for relaxing capacity. 


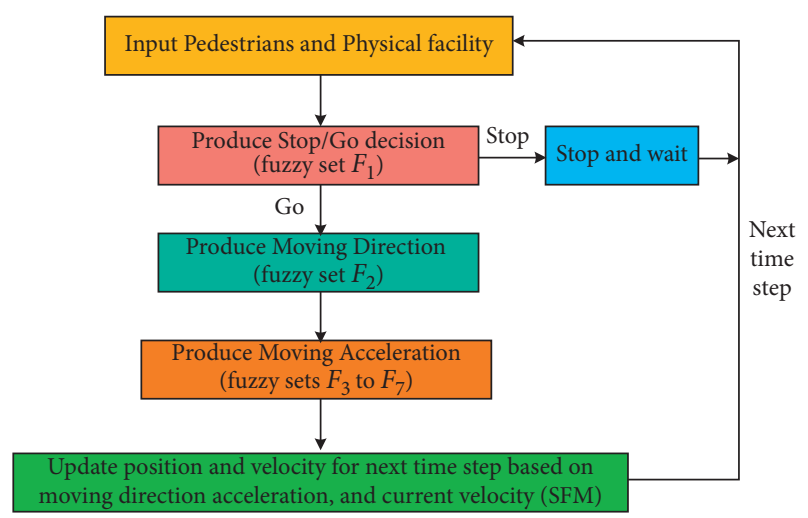

FIgURE 4: The simulation flowchart.

Step 1: fuzzy desired force is divided into several groups according to its number of linguistic terms: \{Strong negative; Negative; Zero; Positive; Strong positive\}. Pedestrian relaxing time can also be divided into three linguistic terms \{Small; Medium; Large\}.

Step 2: relaxing time can be obtained from field observations. Membership function of relaxing capacity is able to be plotted as shown in Figure 5(a): \{Small; Medium; Large\}.

Step 3: when relaxing time is small, relaxing capacity will be slow. So, according to preselection membership functions and fuzzy rules, relaxing capacity can be divided into three ranges, which is shown in Figures 5(b)-5(d). They explain the relationship between relaxing capacity and frequencies of relaxing time. Membership function of relaxing capacity is then calibrated, as shown in Figure 5(e).

Through these three steps, the membership function is selected and calibrated. Similarly, membership function of velocity difference with desired velocity is calibrated:

Step 1: fuzzy desired force is divided into several groups according to its number of linguistic terms: \{Strong negative; Negative; Zero; Positive; Strong positive\}. Velocity differences with desired velocity are divided into \{Very Slow; Slow; Fine; Fast; Very Fast\}.

Step 2: velocity differences with desired velocity can be obtained from field observations. Membership function of velocity difference is able to be plotted.

Step 3: when velocity difference is "Very Slow," the desired force of pedestrian will be "Positive" or "Negative". Therefore, membership functions of velocity difference can be calibrated.

For other fuzzy factors, membership functions are also selected and calibrated by adopting the above same method.

4.2. Comparison between FSMF and ISFM. To verify the proposed fuzzy social force model, the typical classrooms belonging to Chang'an University, Xi'an, China, were selected as the observed site. The size of the observed classrooms is $11 \mathrm{~m} * 9 \mathrm{~m}$. They all have two exits, two exit indicators, and stickers. The simulation scenario is shown in Figure 6. The number of pedestrians in the classroom is 86 , and the distribution of exits and evacuation signs are as shown in Figure 6. In order to obtain more stable simulation results, under the same simulation conditions, 50 experiments were repeated. The velocities of pedestrians were recorded in every experiment. There are 4800 pedestrians totally. The results of one simulation are recorded separately.

Furthermore, the simulation performance of the developed FSFM is tested by comparing with existing ISFM. Figure 7 shows the comparison results of the distribution of all pedestrian simulation speeds in our model and ISFM with the distribution of the observed speeds. As known from Figure 7 , the speed distribution is close to the observed results, which proves the validity of the model. Besides, the mean is $1.49 \mathrm{~m} / \mathrm{s}, 1.56 \mathrm{~m} / \mathrm{s}$, and $1.27 \mathrm{~m} / \mathrm{s}$, respectively, for three results, and the standard deviation is $0.9,1.04$, and 0.73 , respectively. The comparison results show that the simulation results in our model are closer to the actual observed data. The pedestrian speed in ISFM is smaller than actual observed data because, in ISFM, pedestrians cannot use the indicators to find the best movement direction, and they always have to move toward the exits, though the exits cannot be seen under some conditions. When the view of pedestrian is limited, he/she has to slow down until he/she can see the exits. So, the simulation performance of ISFM is worse than that of our model.

As ISFM was developed without considering the evacuation signs, so results from FSMF are compared with results from ISFM through creating the same scenarios with no evacuation signs. That is, evacuation signs are invisible in fuzzy set $F_{6}$. Figure 8 (a) shows the number of pedestrian changes in the classroom by using FSFM and ISFM, and Figure 8(b) shows the velocity-density relationship of FSFM and ISFM. Evacuation time represents the time from the beginning of pedestrian movement to the end of the last pedestrian leaving the classroom. It can be seen from Figure 8 that the results according to FSFM are closer to the observation data. As shown in Figure 8(a), with the evacuation time increasing, pedestrians remaining in the classroom decrease gradually. But the evacuation performance of ISFM is the worst. The evacuation performance of FSFM is close to the actual situation. As shown in Figure 8(b), under the same pedestrian density, the observed speeds are the highest, then the results of FSFM are second, and the results of ISFM are the lowest, which is consistent with the results in Figure 8(a). The proposed model can ensure that the simulation is more realistic and efficient. The model in this paper can be used to access the effectiveness of new pedestrian facilities. But the model needs further study.

However, when the pedestrian density is less than $0.5 \mathrm{ped} / \mathrm{m}^{2}$, the results of FSFM, ISFM, and observation are almost the same because at low density, pedestrians have good vision and enough space to walk, and the interactions between pedestrians are weak. The proposed model makes little sense. But when the density is greater than $0.5 \mathrm{ped} / \mathrm{m}^{2}$, the proposed model helps increase the speed of pedestrian. The higher the crowd density, the more beneficial the 


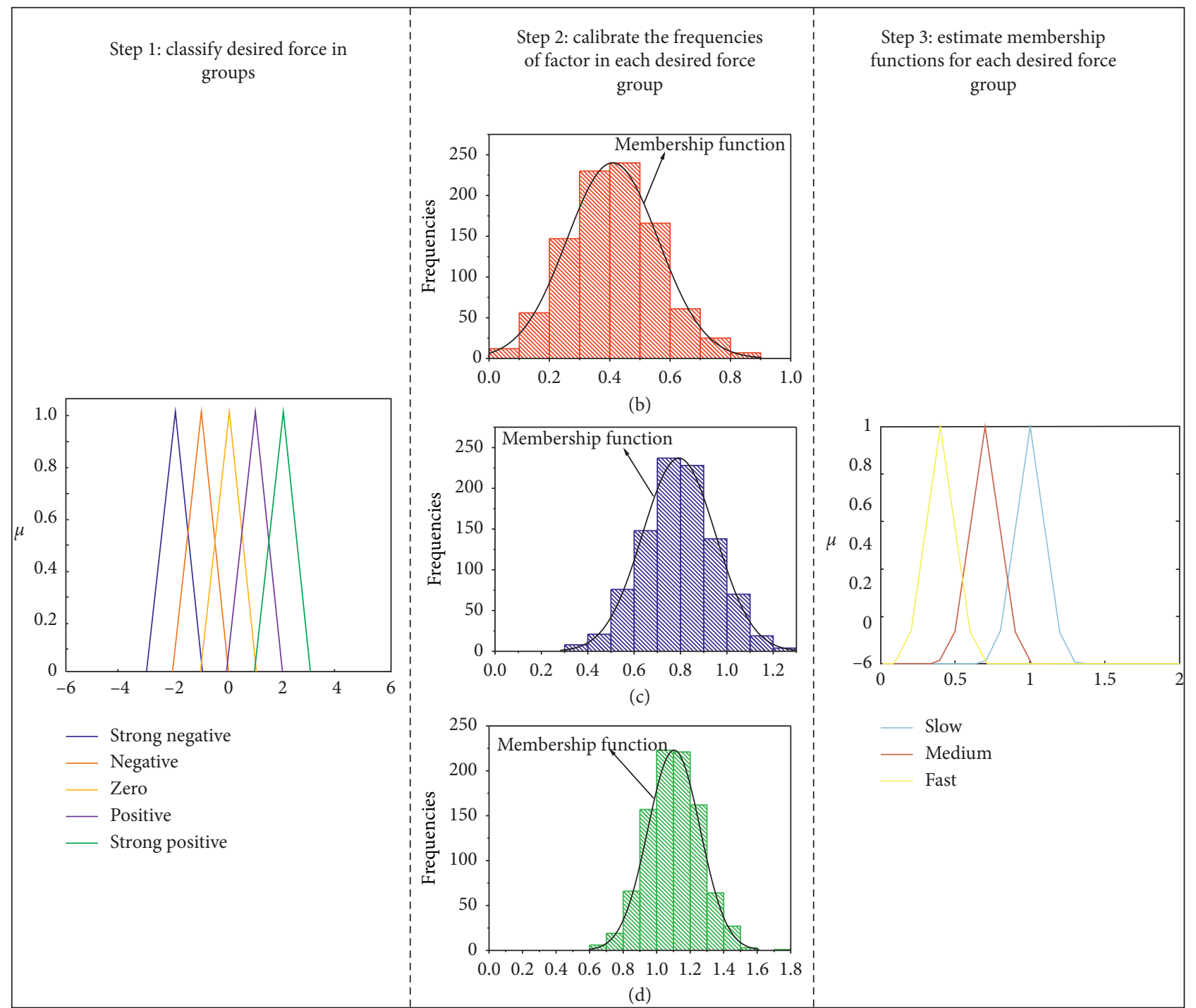

FIGURE 5: Calibration of membership functions (relaxing capacity). (a) Desired force. (b) Relaxing capacity (fast). (c) Relaxing capacity (medium). (d) Relaxing capacity (slow). (e) Relaxing capacity.

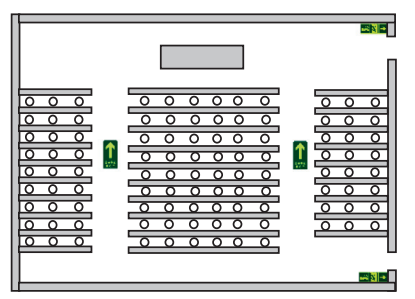

(a)

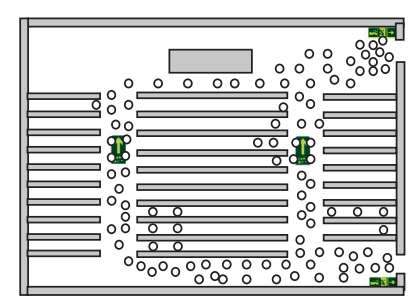

(b)

FIGURE 6: Simulation scenario. (a) Normal. (b) Evacuation.

proposed model is to improve the evacuation efficiency. The simulation results are more consistent with reality.

In order to further verify that the model proposed in this paper is better than the initial social force model, the evacuation efficiency of the crowd is compared. The passing time of pedestrian can be used to reflect the evacuation efficiency of crowd, so the distribution of passing time of 4800 pedestrians was analyzed. As shown in Figure 9, the distribution of passing time obtained from our model is close to the observed results, which proves the validity of the model. Besides, the mean value of passing time obtained from three methods is $36.9 \mathrm{~s}, 23.7 \mathrm{~s}$, and $20.3 \mathrm{~s}$, respectively, and the standard deviation is $14.7,12.3$, and 11.1 , respectively. The comparison results show that the evacuation efficiency of crowd by using 


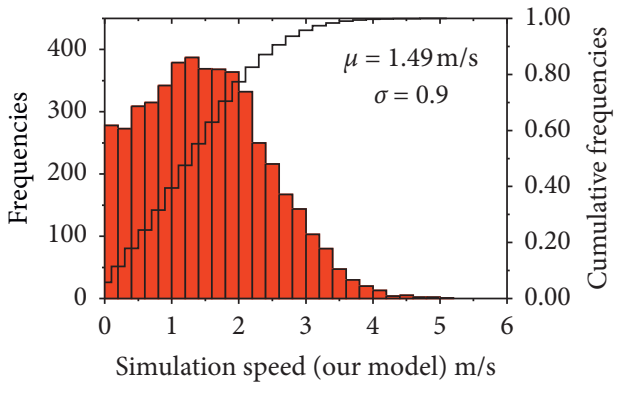

(a)

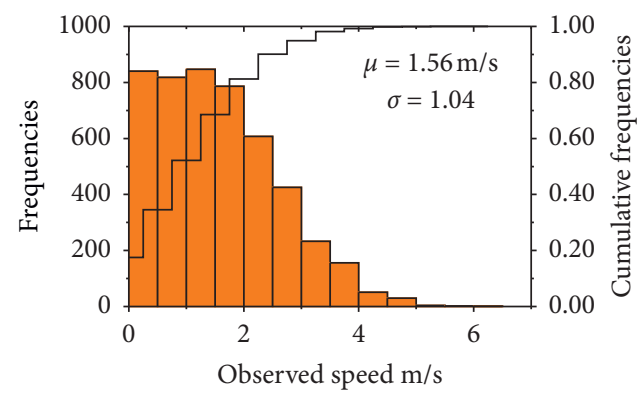

(b)

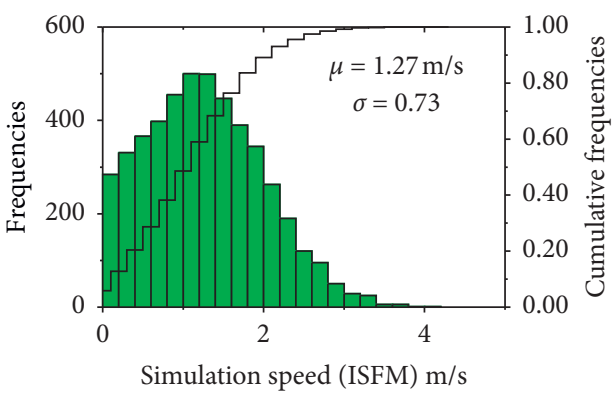

(c)

Figure 7: Comparison of simulated and observed speed.

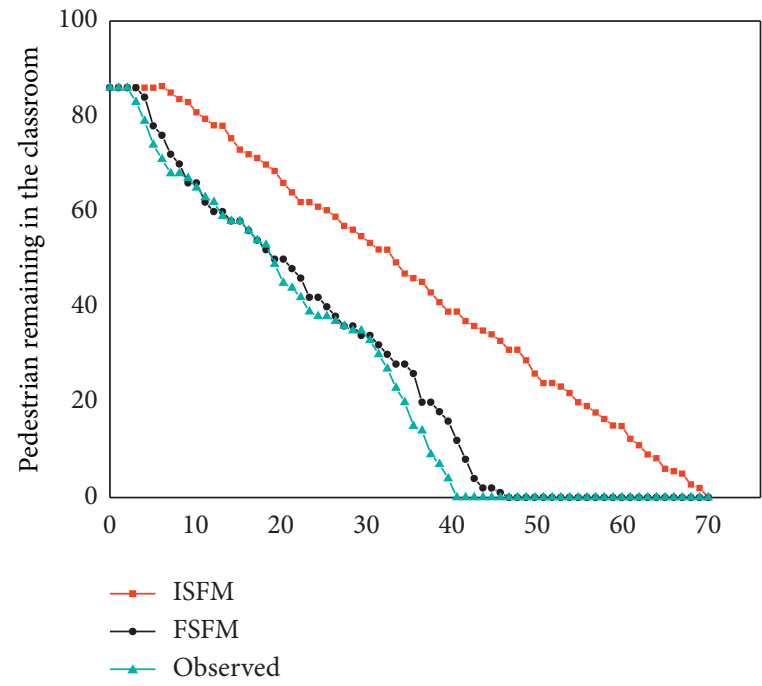

(a)

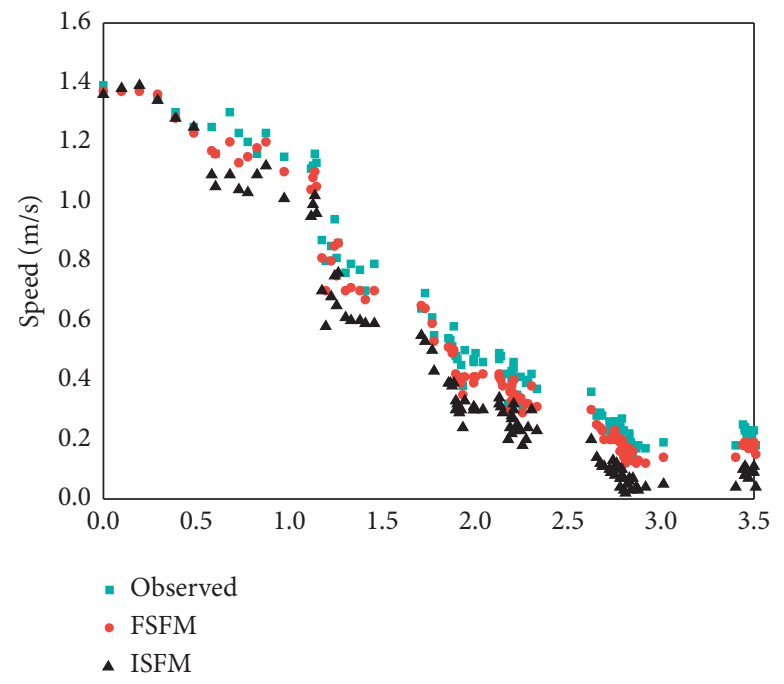

(b)

FIGURE 8: Comparison among FSFM, ISFM, and observation data. (a) Evacuation time. (b) Density.

our model is closer to the actual observed data. The evacuation efficiency of crowd by using ISFM is lower than the actual observed data. This is consistent with the above comparative analysis. In ISFM, although the influences of indicators (exit indicator and exit sticker) are taken into consideration, the influences are quantified as forces. The forces are gravitation, and their directions point to the indicators. When these indicators cannot be seen clearly, pedestrians still move in the directions pointing to the indicators, which may lead to block or stop of pedestrians. However, when these indicators cannot be seen clearly, that is, under view-limited condition, pedestrians can adjust the moving directions moderately according to the vague impression on where are these indicators. In this way, they can avoid blocking or stopping. This kind of fuzzy decision making is one of the advantages of this paper.

\subsection{Model Application}

4.3.1. Number of Exit Stickers and Indicators. Under viewlimited condition, pedestrians cannot judge clearly which directions to move in. At this time, indicators and stickers 


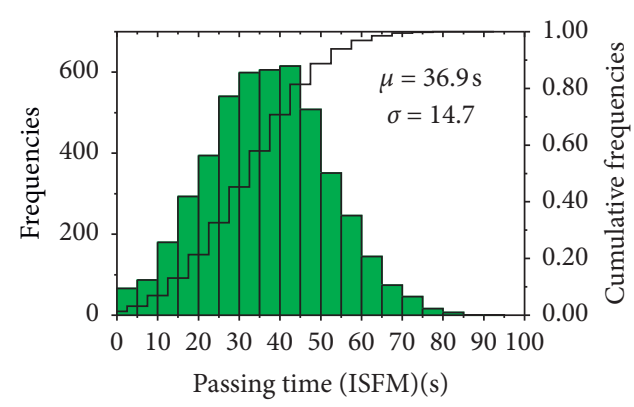

(a)

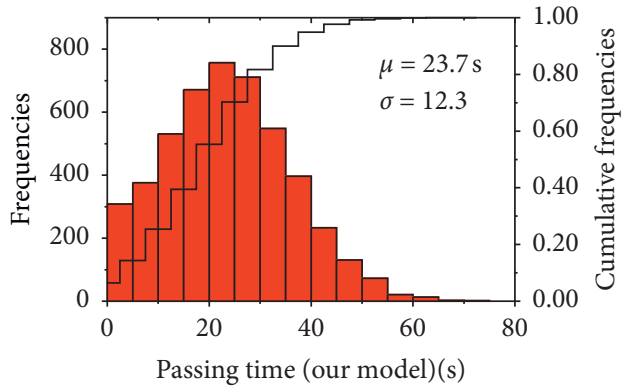

(b)

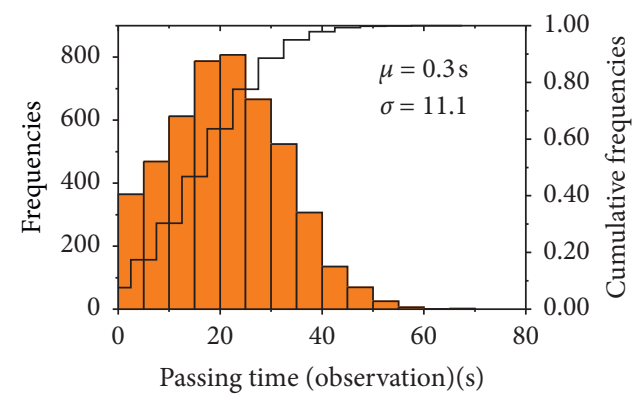

(c)

FIgURE 9: Distribution of passing time.

can help pedestrians to find the best direction of movement. In this way, the efficiency of group evacuation will be greatly improved. Compared with the traditional social force model, fuzzy social force model proposed in this paper can solve this problem. Even if the indicators and stickers cannot be seen clearly, pedestrians can find the best alternative directions of motion in the simulation. In addition, experience shows that the location, number, and spatial distribution of indicators and stickers will affect the evacuation efficiency of the crowd. So, we try to find the best design of indicators and stickers by using the proposed model and verify the model.

Generally speaking, the safety exit indicator should be set on the top of the emergency exit or less than $1 \mathrm{~m}$ from the ground on the wall, and the distance between two indicators should not be more than $20 \mathrm{~m}$. There are two indicators and stickers set up in the classroom, and their locations are shown in Figure 6. As shown in Figure 10, with the increase of indicators and stickers, the downward trend of evacuation time can be roughly divided into two segments: the first segment has a more obvious decline (number $\leq 2$ ). Indicators and stickers can help pedestrians improve evacuation efficiency. After setting two indicators in the classroom, the evacuation time drops from $79 \mathrm{~s}$ to $46 \mathrm{~s}$, and after setting two stickers in the classroom, the evacuation time drops from $72 \mathrm{~s}$ to $46 \mathrm{~s}$. In the first stage, the indicators are more effective than stickers because the indicators are set up above the door, so it is so clear for pedestrians to see and move toward them. In the second stage, obviously, the stickers are more effective than indicators because the indicators are set up within $1 \mathrm{~m}$ to the ground on the wall, so they are blocked by pedestrians ahead and invisible for pedestrians. With the increase of indicators and stickers, the probability of

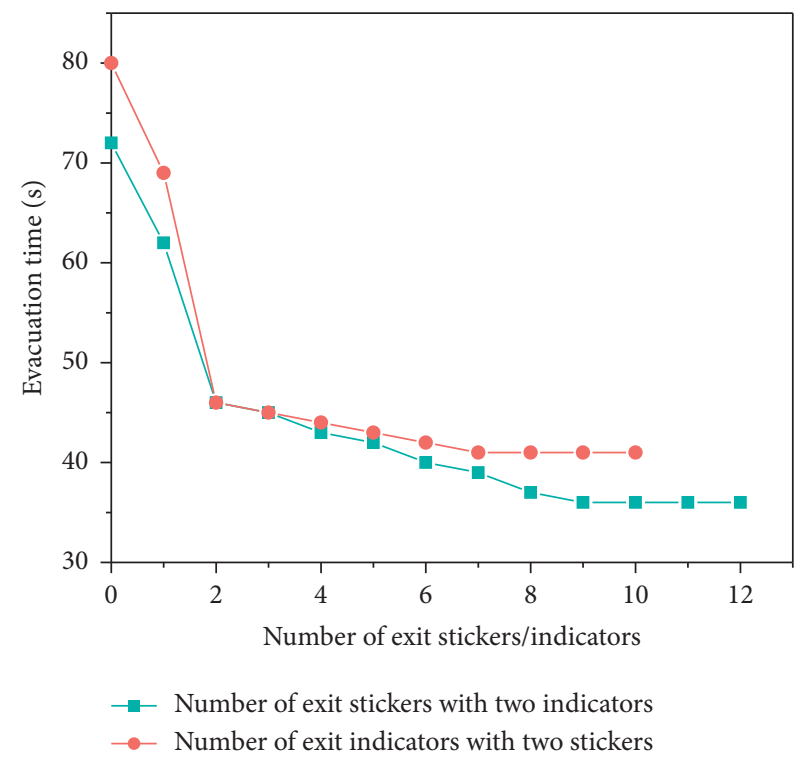

Figure 10: Evacuation time under different conditions.

pedestrians seeing the indicators and stickers increases, so the evacuation turns faster. Therefore, the increase of indicators and stickers in the first stage is most beneficial to the overall evacuation; when the number of indicators and stickers continues to increase to the second stage, the evacuation time will still decline but not so obvious as the first stage; when the number of indicators and stickers is enough, the impact of the number of indicators and stickers on the overall evacuation is very small, and the evacuation time will almost no longer decline. Obviously, in the actual 


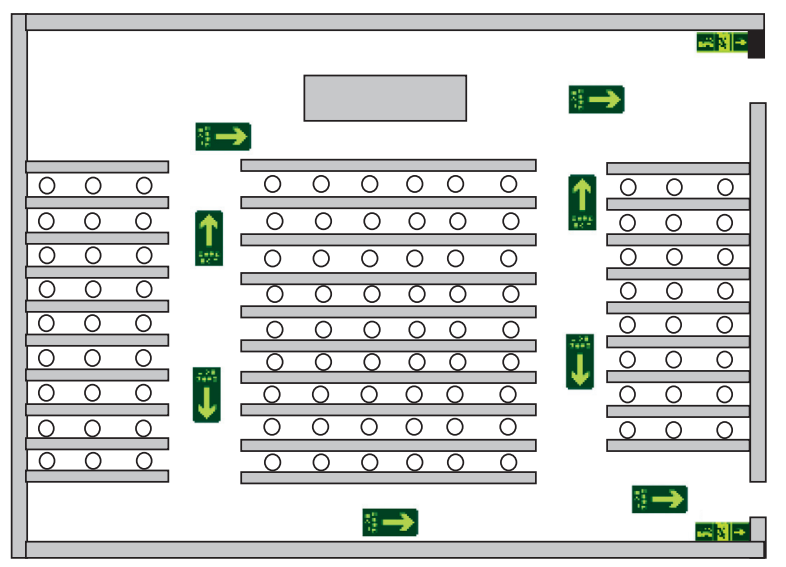

(a)

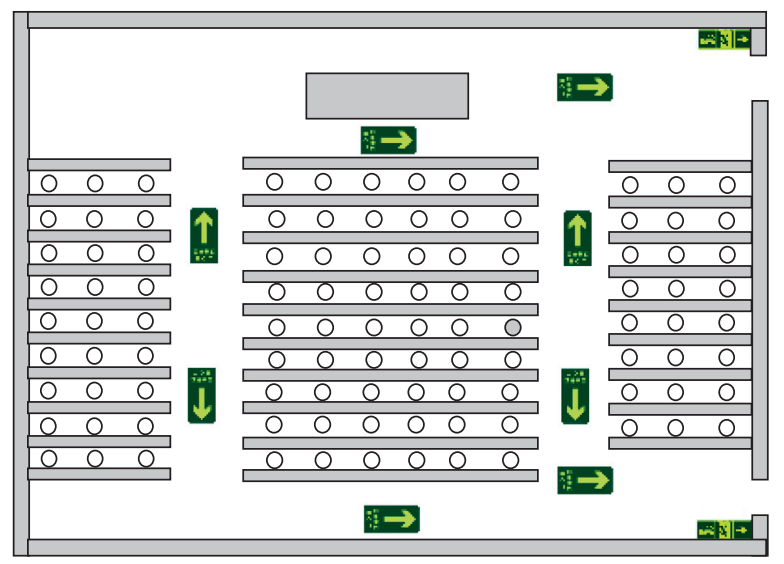

(c)

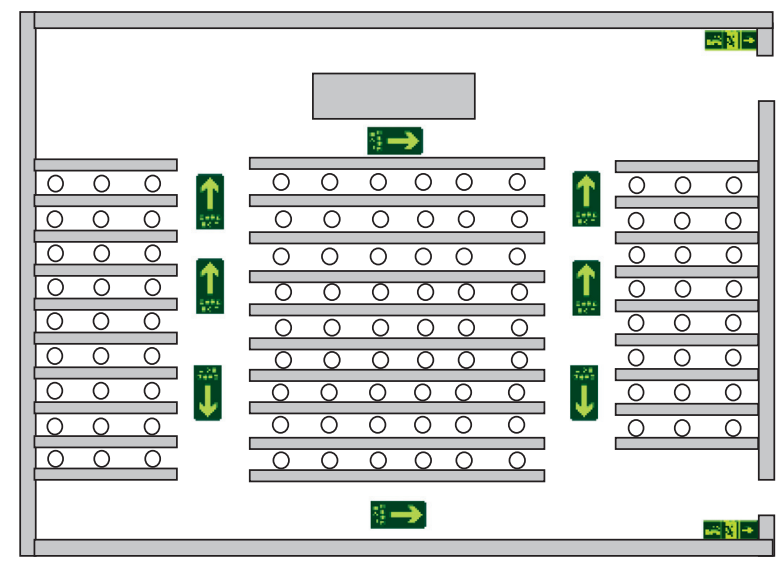

(b)

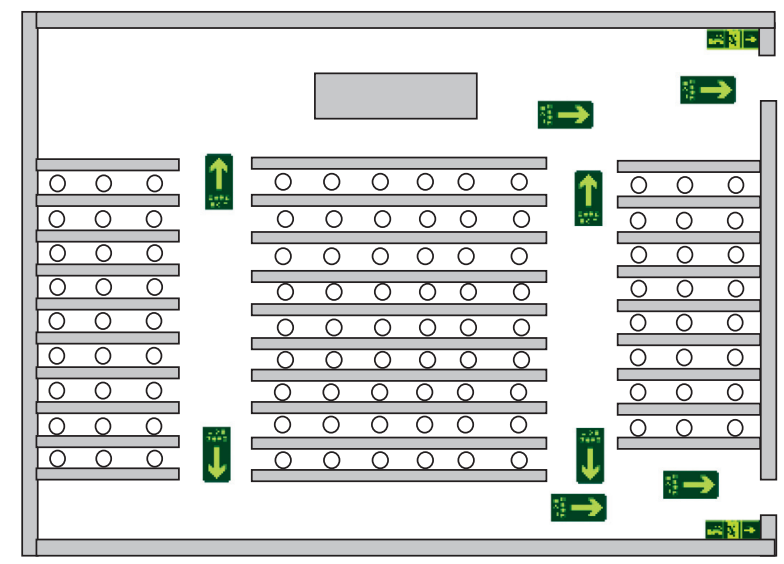

(d)

FIgURE 11: Different space distributions of indicators and stickers. (a) Random distribution. (b) Centralized distribution. (c) Uniform distribution. (d) Exit distribution.

evacuation, the best number of indicators and stickers needs to consider two factors of costs and evacuation time. Therefore, there is a relatively optimal number of indicators and stickers.

4.3.2. Distribution of Exit Stickers and Indicators. The number of exit stickers and indicators influences the evacuation time. Besides, the space distribution of indicators and stickers also affects the evacuation time. The evacuation time under different space distribution of indicators and stickers is compared. Figure 11 shows four different distributions of stickers: (a) random distribution, (b) centralized distribution, (c) uniform distribution, and (d) exit distribution. It should be noted that all pedestrians are randomly distributed in the classroom.

The simulation results are shown in Figure 12. It is found that the average evacuation time is the shortest under the condition of uniform distribution of stickers. Compared with other distributions, the uniform distribution of stickers can cover a larger guiding area, so more pedestrians can see stickers and move in the directions of indications. It should be noted that pedestrians are randomly distributed in the above simulations. If pedestrians belong to specific

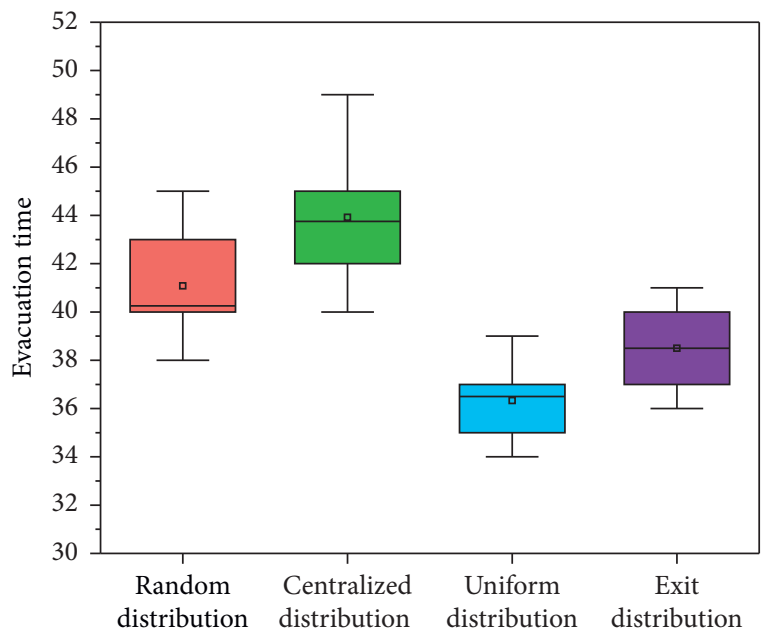

Figure 12: Evacuation time under different distribution conditions.

distribution, the results will be different. But even so, the proposed model can still find a relatively better distribution of stickers through simulation. 


\section{Conclusions}

Pedestrian behavior is very flexible and vague, and fuzzy logic model can reflect these characteristics. So, FSFM, which incorporates fuzzy logic and social force model (SFM), is proposed to simulate pedestrian evacuation in classroom. Simulation results show that the proposed model can improve ISFM by using fuzzy rules to model the movements and decision-making behavior of pedestrians. Seven fuzzy sets are designed to simulate movements of pedestrians, including stop/go, moving direction, desired force, force from obstacles, force from pedestrian, force from indicators, and acceleration. Subsets at different zones are introduced to represent dynamic influences of indicators and stickers, neighboring pedestrians, and tables/chairs on each pedestrian. This developed model has the advantages of SFM and fuzzy logic. It is clear that FSFM avoids the heavy parameter calibration task and unnecessary complexity of the calculation process. Simulation results show the proposed model can obtain results similar to observed data, such as speed, passing time, and the relationship between velocity and density. Besides, the proposed model is able to be used to verify the effectiveness of the new facility and get the best design through multiple simulation experiments.

The developed FSFM is able to help managers reproduce the evacuation process of students in classroom under various facilities conditions. The microscopic simulation model provides a convenient and effective tool to estimate pedestrian movement, and the impacts of new facilities. Some improvements of this model refine and extend the applications of developed FSFM in future work. Compared to SFM, contributions of the FSFM can be summarized as follows: firstly, FSFM can explain the complex and vague interaction process in a realistic and straightforward method because linguistic terms are very suitable for describing input factors; secondly, compared to the mathematical social force model, FSFM allows to select the membership functions according to the observed data; thirdly, the simulation results obtained by the proposed model are closer to the real observation data. Last but not least, FSFM reduces the calculation of the initial model.

However, the model can be improved from the following aspects. Firstly, movement experiments (based on virtual reality simulation) and questionnaire will be adopted to acquire physiological cognition data of pedestrians. Secondly, other factors, such as age and gender, which can affect the behaviors of pedestrians, should be taken into consideration. Finally, some pedestrians are more risk-taking than others. This characteristic affects stop/go decision and reaction of neighboring pedestrians.

\section{Data Availability}

The raw/processed data required to reproduce these findings cannot be shared at this time as the data also form part of an ongoing study.

\section{Conflicts of Interest}

The authors declare that they have no conflicts of interest.

\section{Acknowledgments}

This research was supported by Fundamental Research Funds for the Central Universities (no. 300102239103) and Young Teachers Research Project for Xi'an University of Technology (no. 256081921).

\section{References}

[1] L. Luo, Z. Fu, H. Cheng, and L. Yang, "Update schemes of multi-velocity floor field cellular automaton for pedestrian dynamics," Physica A: Statistical Mechanics and Its Applications, vol. 491, pp. 946-963, 2018.

[2] Q. Wang, H. Dong, B. Ning, L. Y. Wang, and G. Yin, "Twotime-scale hybrid traffic models for pedestrian crowds," IEEE Transactions on Intelligent Transportation Systems, vol. 19, no. 11, pp. 3449-3460, 2018.

[3] L. F. Henderson, "The statistics of crowd fluids," Nature, vol. 229, no. 5284, pp. 381-383, 1971.

[4] R. L. Hughes, "A continuum theory for the flow of pedestrians," Transportation Research Part B: Methodological, vol. 36, no. 6, pp. 507-535, 2002.

[5] N. K. Mahato, A. Klar, and S. Tiwari, "Modeling and simulation of macroscopic pedestrian flow models," Progress in Industrial Mathematics at ECMI 2018, pp. 437-444, Springer, Berlin, Germany, 2019.

[6] S. P. Hoogendoorn, F. van Wageningen-Kessels, W. Daamen, D. C. Duives, and M. Sarvi, "Continuum theory for pedestrian traffic flow: local route choice modelling and its implications," Transportation Research Part C: Emerging Technologies, vol. 59, pp. 183-197, 2015.

[7] S. Bandini, M. Mondini, and G. Vizzari, "Modelling negative interactions among pedestrians in high density situations," Transportation Research Part C: Emerging Technologies, vol. 40, pp. 251-270, 2014.

[8] J. Yang, Z. S. Hou, and M. H. Zhang, "Simulation of pedestrian dynamic using a Vector Floor 44 Field model," International Journal of Modern Physics C, vol. 24, no. 4, 2013.

[9] B. Leng, J. Wang, W. Zhao, and Z. Xiong, “An extended floor field model based on regular hexagonal cells for pedestrian simulation," Physica A: Statistical Mechanics and Its Applications, vol. 402, pp. 119-133, 2014.

[10] T.-Q. Tang, Y.-X. Shao, and L. Chen, "Modeling pedestrian movement at the hall of high-speed railway station during the check-in process," Physica A: Statistical Mechanics and Its Applications, vol. 467, pp. 157-166, 2017.

[11] X. Zhou, P. Wang, and X. Jia, Cellular Automaton Simulation of Pedestrian Evacuation Considering the Guidance information, The National Academies of Sciences, Engineering, and Medicine, Washington, DC, USA, 2017.

[12] C. Feliciani and K. Nishinari, "An improved Cellular Automata model to simulate the behavior of high density crowd and validation by experimental data," Physica A: Statistical Mechanics and Its Applications, vol. 451, pp. 135148, 2016. 
[13] L. Fu, W. Song, and S. Lo, "A fuzzy-theory-based behavioral model for studying pedestrian evacuation from a single-exit room," Physics Letters A, vol. 380, no. 34, pp. 2619-2627, 2016.

[14] M. Zhou, H. Dong, D. Wen, X. Yao, and X. Sun, "Modeling of crowd evacuation with assailants via a fuzzy logic approach," IEEE Transactions on Intelligent Transportation Systems, vol. 17, no. 9, pp. 2395-2407, 2016.

[15] T. Liu, X. Yang, Q. Wang, M. Zhou, and S. Xia, "A fuzzytheory-based cellular automata model for pedestrian evacuation from a multiple-exit room," IEEE Access, vol. 8, pp. 106334-106345, 2020.

[16] X. Yang, X. Yang, and Q. Wang, "Pedestrian evacuation under guides in a multiple-exit room via the fuzzy logic method," Communications in Nonlinear Science and Numerical Simulation, vol. 83, p. 105138, 2020.

[17] D. Helbing and P. Molnár, "Social force model for pedestrian dynamics," Physical Review E, vol. 51, no. 5, p. 4282, 1995.

[18] H. Xi, Y. J. Son, and S. Lee, "An integrated pedestrian behavior model based on extended decision field theory and social force model," in Proceedings of the 2010 Winter Simulation Conference, pp. 824-836, Baltimore, MD, USA, December 2010.

[19] J. K. K. Yuen and E. W. M. Lee, "The effect of overtaking behavior on unidirectional pedestrian flow," Safety Science, vol. 50, no. 8, pp. 1704-1714, 2012.

[20] T. I. Lakoba, D. J. Kaup, and N. M. Finkelstein, "Modifications of the helbing-molnár-farkas-vicsek social force model for pedestrian evolution," Simulation, vol. 81, no. 5, pp. 339-352, 2005.

[21] D. R. Parisi, M. Gilman, and H. Moldovan, "A modification of the Social Force Model can reproduce experimental data of pedestrian flows in normal conditions," Physica A: Statistical Mechanics and Its Applications, vol. 388, no. 17, pp. 36003608, 2009.

[22] S. Xu and B. L. Duh, "A simulation of bonding effects and their impacts on pedestrian dynamics," IEEE Transactions on Intelligent Transportation Systems, vol. 11, no. 1, pp. 153-161, 2010.

[23] F. Johansson, A. Peterson, and A. Tapani, "Waiting pedestrians in the social force model," Physica A: Statistical Mechanics and Its Applications, vol. 419, pp. 95-107, 2015.

[24] Y. Han and H. Liu, "Modified social force model based on information transmission toward crowd evacuation simulation," Physica A: Statistical Mechanics and Its Applications, vol. 469, pp. 499-509, 2017.

[25] L. A. Zadeh, "Fuzzy sets," Information and Control, vol. 8, no. 3, pp. 338-353, 1965.

[26] D. Dubois and H. Prade, "The three semantics of fuzzy sets," Fuzzy Sets and Systems, vol. 90, no. 2, pp. 141-150, 1997.

[27] M. Nasir, S. Nahavandi, and D. Creighton, "Fuzzy simulation of pedestrian walking path considering local environmental stimuli," in Proceedings of the 2012 IEEE International Conference on Fuzzy Systems, pp. 1-6, IEEE, Brisbane, Australia, June 2012.

[28] M. Nasir, C. P. Lim, S. Nahavandi, and D. Creighton, "A genetic fuzzy system to model pedestrian walking path in a built environment," Simulation Modelling Practice and Theory, vol. 45, pp. 18-34, 2014.

[29] C. Chai, Y. D. Wong, M. J. Er, and E. T. M. Gwee, "Fuzzy cellular automata models for crowd movement dynamics at signalized pedestrian crossings," Transportation Research Record: Journal of the Transportation Research Board, vol. 2490, no. 1, pp. 21-31, 2015. 